\title{
$\mathrm{UN}$ 학교재난안전 고찰
}

박 윤 주

한국교원대학교

\section{A Review of the United Nations' School Disaster Safety}

\author{
Youn-Ju Park \\ Korean National University of Education
}

\begin{abstract}
Purpose: This review aims to provide an understanding of the $U N$ recommendations regarding school disaster safety in the context of Korea's school safety system. Methods: Relevant literature and reports on the UN's school disaster safety were investigated. On basis of the analysis, this review closely examined how the UN school disaster safety had been developed and what it had proposed. Results: Major findings from this review regarding the UN school disaster safety were to: 1) utilize "all-hazards approach"; 2) focus on "community-based approach"; 3) explore "education for sustainable development"; and 4) emphasize "informal disaster safety education." Conclusion: This review on the UN school disaster safety may give a critical implication to Korea's school safety education.
\end{abstract}

Key Words: The UN school disaster safety, Disaster safety education, School disaster safety, Disaster risk reduction

\section{서 론}

\section{1. 연구의 필요성}

전 세계적으로 2000년에서 2015년까지 5,900건 이상의 재 난이 발생하였으며, 재난으로 120 만명이 사망하고, 32 억명이 피해를 입었다. 또한 이로 인한 경제적 손실은 2조불에 달하여, 1990년대 6천억불에 비하여 빠른 속도로 증가하고 있음을 보 여주고 있다. 이러한 재난의 심각한 피해로 인해 UN 기구들은 재난안전을 주요 전략으로 선정하고, 재난안전 활동을 강화하 고 있다. 대표적인 예로 재난안전과 관련된 12 개 UN 기구의 2014 2017년 실행 전략을 살펴보면 종전 대비 70\% 이상 관련 사업의 비중을 확대하고 있다[1].

UN은 "재난안전을 위한 Hyogo 행동계획(Hyogo Frame- work for Action 2005 2015: Building the Resilience of Nation and Communities to Disaster)"에서 재난안전에 있어 학교교육의 중요성을 강조하면서 “재난안전은 학교로부터 (Disaster Risk Reduction Begins at School)"라는 기치 하에, 학생들을 재난안전의 “변화의 주체(agent of change)"로 교 육시켜야 된다고 제시하였다. 또한 학교는 재난안전 지식과 기술을 효과적으로 공유하고 보급시킬 수 있는 대표적 기관으 로서 학교재난안전교육은 가정과 사회로의 확산 뿐 만 아니 라, 미래세대의 안전을 확보한다는 측면에서 매우 중요하다고 강조하고 있다[2]. 현재 세계 각국에서는 이러한 UN의 재난안 전의 관점을 반영하여 학교재난안전교육에 범국가적인 정책 역량을 집중하고 있다.

최근 우리나라는 인적재난 및 자연재해를 수차례 겪는 과정 에서 국가 차원의 재난안전 대응 역량에 있어 심각한 한계를

\footnotetext{
Corresponding author: Youn Ju Park

Korean National University of Education, 250 Taeseongtabyeon-ro, Gangnae-myeon, Heungdeok-gu, Cheongju 28173, Korea.

Tel: +82-43-230-3406, Fax: +82-43-230-3401, E-mail: 20green@knue.ac.kr
}

Received: Nov 1, 2016 / Revised: Nov 18, 2016 / Accepted: Nov 22, 2016

This is an open access article distributed under the terms of the Creative Commons Attribution Non-Commercial License (http://creativecommons.org/licenses/ by-nc/3.0), which permits unrestricted non-commercial use, distribution, and reproduction in any medium, provided the original work is properly cited. 
드러냈다. 특히, 2015년 국가시스템을 마비시킨 메르스 사태, 2016년 9월 발생한 규모 5.8의 경주 지진 등 연속적인 대형재 난으로 우리나라가 더 이상 재난의 안전지대가 아니라는 위기 인식이 확산되고 있다. 현재 우리나라는 국가차원의 재난안전 역량 강화를 위한 효과적 방안 마련이 시급하다. 이러한 시점 에서 재난안전에 대한 근본적인 재검토가 요구되고, 이를 통 해 새로운 학교재난안전교육의 틀을 갖추어야 할 필요가 있다.

본 연구에서는 UN 재난안전 개념과 재난안전 대응이 어떤 내용으로 발전되었는지를 살펴보고, 학교재난안전의 주요 내 용과 특징에 초점을 두고 UN 발간 자료 및 학술논문 등을 기 초로 분석하였다. 본 연구에서 분석한 $\mathrm{UN}$ 학교재난안전은 최 근 학교 재난안전교육에 대한 관심과 요구가 높아지고 있는 우 리나라의 학교재난안전교육 정책 수립을 위한 기초자료와 시 사를 제공하고자 하였다.

\section{UN 재난안전 개념 및 발전}

\section{UN 재난안전 개념}

$\mathrm{WHO}$ [3]는 재난을 "한 지역사회가 감당할 수 없는 고통을 초래하며, 정상적 생존 환경을 혼란시키는 사건”으로 정의하 여 재난의 핵심요소로서 지역사회의 심각한 피해와 이를 초래 하는 사건 등 2가지 요소를 제시하였다. 이후 UNISDR [2]은 재난을 "지역사회의 대응역량을 넘어서 지역사회의 인적, 물 적, 경제적, 환경적 자원이 기능적으로 심각하게 훼손된 상태" 로 정의하면서 지역사회의 재난대응역량을 강조하는 개념으 로 발전하였다.

한편 재난의 유형은 발생 원인에 따라 다양하게 분류되나, 일 반적으로 자연재난과 인적재난으로 구분하고 있다. 그러나 자연 재난의 용어와 관련하여 많은 논란이 있어 왔다. Alexander [4] 는 “자연재난" 은 환경적 속성인 “자연"과 인간의 취약성을 나타 내는 “재난"을 혼용한 잘못된 용어라고 지적하였다. 이와 유사 한 관점으로 Basher [5]는 자연은 통제 불가능하지만, 재난은 통 제 가능한 개념으로 두 용어를 함께 사용한 “자연재난" 은 “모순 용어(oxymoron)"로서, “자연재난" 대신 “재난”으로 대체되 어야 한다는 의견을 제시하였다. 이와 같이 자연재난이라는 용 어에는 인간의 능력과 한계를 벗어난 영역으로 사전 관리가 불 가능하다는 의미가 포함되어 있다. 이러한 측면에서 종전 재난 안전은 재난 발생 후 사후 대응에 초점을 두어, 정부 및 지역사 회의 긴급구호 활동 등을 주요대상으로 하였다. 자연재난의 용 어에 대한 논란은 2005년 마련된 Hyogo 행동계획에 반영되어
종전 “자연재난"에서 “자연”을 삭제한 “재난”으로 사용함으로 써 정부의 적극적 역할이 강조되기 시작하였다. 이후 $\mathrm{UN}$ 의 재 난안전은 “자연위험사건(natural hazard)"이 "재난(disaster)" 으로 발전하는 것을 방지하는 사전적 대응의 개념으로 자리 잡 고 있다.

이와 같이 재난의 사전 대응 개념과 함께 재난에 대한 국가 의 역할이 강조되면서 재난의 원인으로서 "위험(risk)"에 대한 연구가 많은 발전을 거두어왔다. 대표적인 연구로 Ansell과 Wharton [6]은 현대 위험측정의 기본모델로 널리 활용되고 있 는 "위험(risk)=가능성(likelihood) $\times$ 결과(consequences)" 공 식을 통하여 재난 위험의 발생가능성 또는 잠재적 결과를 감소 시킨다면 재난에 효과적으로 대응할 수 있음을 제시하였다. 이 러한 재난위험의 개념을 반영하여 WHO [3]는 "재난(disaster)= 취약성(vulnerability) + 위험사건(hazard)"의 공식을 제시함 으로써 국가 및 지역사회의 취약성과 위험사건의 발생빈도가 재난 피해를 결정한다고 밝혔다. 또한 취약성을 “재난으로 인 해 신체적, 사회적, 경제적, 환경적 측면에서 지역사회가 영향 을 받는 정도”로 정의하고, 지역사회가 취약할수록 재난으로 인한 피해가 크다는 것을 강조하였다. 따라서 재난으로부터 피해를 최소화하기 위해서는 취약성을 감소시키기 위한 국가, 지역사회, 기관 및 개인의 역량 강화가 필요함을 언급하고 있다. 이러한 역량 강화의 필요성을 직접적으로 반영하여 UNISDR [2]은 "위험(risk)=위험사건(hazard) $\times$ 취약성 (vulnerability)/ 역량(capacity)"라는 공식을 통해 위험사건 및 취약성에 대한 대 응 역량이 재난 위험정도를 결정하는데 주요한 역할을 한다고 제시하였다. 위에서 언급한 공식들은 다음과 같이 재난안전의 세 가지 실천적 측면을 강조하는 것으로 요약할 수 있다: 1) 재 난으로 발전될 가능성이 높은 위험요소의 조기 발견, 2) 국가, 지역사회, 조직에 내재하고 있는 취약성 규명 및 제거 노력, 3) 재난 발생 후 피해를 최소화하고 회복하기 위한 정부·지역사 회· 조직·개인의 역량 강화.

최근 국가 및 지역사회의 재난 대응 역량과 관련하여 “재 난 회복탄력성(disaster resilience)"의 개념이 강조되고 있 다. Hyogo 행동계획은 재난 회복탄력성을 "개인 · 기관·지역 사회 및 정부 차원에서의 재난 회복 능력"으로 정의하고 각 주체의 재난 학습능력에 따라 좌우되며, 미래의 위험을 감소 시키도록 한다고 강조하였다[7]. 이러한 측면에서 재난 회복 탄력성은 취약성과 반대적인 개념으로 사용되어지기도 하나, 일반적으로 취약성은 위험요소인 반면, 재난 회복탄력성은 대 응능력의 요소로 언급되어지고 있다. 이러한 관점을 반영하여 $\mathrm{UNISDR}$ 은 재난위험이 많은 도시 지역의 재난 회복탄력성 증 
진에 초점을 둔 "도시위험저감 및 회복탄력성 프로그램(Urban RiskReduction and Resilience)”을 수립하고, 2010년부터 “회복 탄력적인 도시 만들기 캠페인(Making Cities Resilient Campaign)"을 전개하여, 전 세계적으로 119 개국 3천여 도시가 가입하고 있으며, 2015년에만 500개 도시가 신규 등록하였다. 또한 UNICEF를 중심으로 학교에서의 재난안전 회복능력을 강 조하고 이를 증진하기 위한 회복교육(resilience education)을 학교재난안전교육의 핵심내용으로 제시하였다[8].

위에서 살펴본 바와같이 $\mathrm{UN}$ 의 재난안전 개념은 크게 사전대 응(proactive)차원의 "재난위험저감(Disaster Risk Reduction: $\mathrm{DRR}$ )"과 효과적인 사후 대응(reactive)을 위한 “회복탄력성 (resilience)”등 2가지 측면으로 요약할 수 있다.

\section{UN 재난안전 발전}

1980년대 들어 기후변화 등의 영향으로 전 세계적으로 재난 피해가 보다 심각해질 것으로 예상되면서 재난대응은 국가 간 공동 협력 과제라는 공감대가 형성되기 시작하였다. 이러한 배 경으로 UN은 1990년대를 “자연재난 저감을 위한 10년(the International Decade for Natural Disaster Reduction: IDNDR)"으로 정하고 재난안전을 위한 적극적인 활동을 본격 적으로 전개하였다. 이후 1999년 IDNDR이 종료 됨에 따라 "재난 저감을 위한 국제전략(the International Strategy for Disaster Reduction, ISDR)"을 후속적으로 마련하였다. ISDR 은 재난안전의 비전을 재난예방 문화 확산을 통한 사전예방으 로 전환하고, 실천 전략으로 정부의 재난저감 책무 및 지역사 회의 재난 대응능력을 증진시켜야 한다고 밝혔다.

21세기에 들어서면서 재난이 대형화, 복합화 및 국제화되 는 추세로 범세계적 차원에서 공동 연구하고, 대응할 필요성 이 강화되었으며, 재난위험을 사전에 경감하는 것이 재난안전 의 핵심으로 자리잡았다. 이러한 내용을 반영하여 2005년 마 련된 Hyogo 행동계획은 5 개 재난위험저감의 우선 과제로 1) 재난안전 거버넌스 및 정책, 2) 재난안전 위험 규명 및 조기 경 고, 3) 재난위험저감 지식, 혁신 및 교육 활용, 4) 재난위험요소 경감, 5) 재난대비 강화 등을 제시하였다. 특히 재난 취약 계층 보호를 위한 국가 재난관리체계를 강조하고, 국제 재난안전 협 력네트워크가 강화되는 계기를 마련하였다[7].

Hyogo 행동계획이 2015년 종료됨에 따라 UN 회원국들은 "재난위험저감을 위한 Sendai 계획(the Sendai Framework for Disaster Risk Reduction 2015 2030)"을 합의하였다. Sendai 계획은 지난 10 년 동안 약 70 만명의 사망자를 발생시
키고, 경제적 손실 또한 1 조 3 천억불에 달하는 등 오히려 재난 피해가 급증하였음을 지적하고, 1) 재난예방을 위한 국제협력 강화, 2) “재난 전보다 나은 복구(Build Back Better)", 3) 취약 계층을 위한 공중보건 및 교육 지원, 4 ) 지역사회 재난 대응 역 량 강화, 5) 재난감소 목표 설정 등의 필요성을 보완 제시하였 다. 이러한 목적 달성을 위하여 UNISDR은 "7년 동안 달성할 7가지의 목표(7 Targets, 7 Years)"를 수립하여, 2016년 재난 사망률 감소, 2017년 재난피해자 감소, 2018년 재난 경제적 손 실 감소, 2019년 재난 회복탄력성을 위한 역량개발 및 건강과 교 육시설 기반 구축, 2020년 재난위험저감 도입 국가 및 도시 증 가, 2021년 개발도상국 지속 지원을 통한 국제협력 강화, 2022 년 복합재난의 조기경보와 재난위험정보 제공 확대 등을 언급 하고 있다. 2016년 목표인 재난사망률 감소를 위한 실천 전략으 로 "국제 재난저감의 날(the International Day for Disaster Reduction)"의 캠페인 주제를 “Live to Tell”로 정하고, 재난에 서 가장 중요한 것은 재난으로부터 살아남는 것이라는 점을 강 조하고 있다[9].

\section{UN 학교재난안전 분석}

\section{UN 학교재난안전의 주요내용}

일반적으로 학교재난안전은 "학생을 포함한 학교 전체 구 성원으로 하여금 재난 발생 시 어떠한 혼란, 위해 및 위험이 없 도록 하기 위한 학교교육 활동으로 효과적인 학습 환경을 조성 하기 위한 노력"으로 규정할 수 있다. Hyogo 행동계획은 학교 재난안전의 목적을 1 ) 재난위험저감의 지속발전 교육정책 통 합, 2) 국가 및 지역사회의 회복역량 강화, 3) 재난위험저감의 학교재난안전 프로그램 통합 등 3가지로 제시하였다. 이러한 목 적을 반영하여 UNICEF 등[10]은 학교재난안전의 기본틀을 "통 합적 학교안전(Comprehensive School Safety)” 모형으로 설명 하고, 학교재난안전 관리, 안전한 학습시설, 위험저감 및 회복 교 육 등 3개 핵심요소로 설명하고 있다(Figure 1).

첫째, 학교재난안전 관리는 재난 발생 시 학생과 교직원을 보호하고, 학교 혼란을 최소화하여 교육지속성을 보장하며, 궁극적으로는 학교 안전문화를 증진시키는데 목적이 있다. Petal [11]은 성공적 학교재난안전 관리를 위해서는 학교 전체 구성원, 가정 및 지역사회 등이 참여하는 지속적인 체계를 구 축하고, 이를 통해 학교 위험저감 계획 수립, 학교 및 지역사회 위험측정, 학교 위험저감 활동, 학교재난안전 대응역량 개발, 위험저감 계획 평가 등을 주요 내용으로 다루어야 한다고 제시 


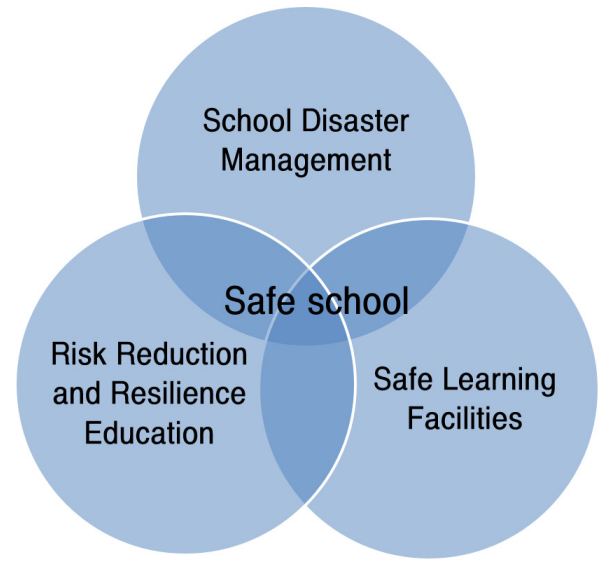

UNICEF et al. 2013[10]

Figure 1. Comprehensive school safety.

하였다.

둘째, 안전한 학습시설을 위하여 학교시설의 위험관리를 강 조하고, 안전한 학교건물 건축과 학교시설에 대한 안전관리 측면으로 구분하였다. 특히, 시설 안전관리 측면에서는 구조 적 안전과 비구조적 안전으로 유형화하여, 구조적 안전은 지 진 등 재난 내구성에 대한 진단 및 관리에 중점을 두며, 비구조 적 안전은 가연성 물질 제거, 비상출입구 방해물 제거 등의 활 동에 초점을 두고 있다. 이러한 $\mathrm{UN}$ 의 안전한 학교시설 관점을 반영하여 “위기대응교육네트워크(the Inter-agency Network for Education in Emergencies)" 는 안전한 학습시설에 대한 최소기준을 다음과 같이 제시하였다: 1) 안전한 곳에 학교배치 및 재난대비 설계; 2) 안전하지 못한 학교 시설의 우선 재배치 및 보수; 3) 구조적 학교시설 위험 최소화; 4) 학교 설계에 무장 애(barrier free) 및 안전요소 반영; 5) 재난 시 학교교육 지속성 및 재난대피 시설 확보; 6) 학교통학로 위험요소 제거; 7) 위생 적인 급수 등 친환경 시설 설치; 8) 학교안전계획 수립 및 모니 터링 등[12]

셋째, 재난위험저감 및 회복교육의 강화로서 재난안전교육 을 학교 정규교육과정에 반영할 것을 권고하고, 재난안전교육 을 강화하여 학생들의 안전을 제고하고, 지역사회의 학교재난 안전에 대한 관심을 높여야 한다고 강조하였다. 이를 위한 학교 재난안전 교육의 기본 틀로서 “KIDA 모델”을 설정하고, 지식 (knowledge), 흥미(interest), 욕구(desire) 및 행동(action) 등 4 가지 핵심요소를 제시하였다. 지식, 흥미 및 욕구 등 3 개 요소 는 행동을 증진시키기 위한 투입요소로서 학교재난안전 교육 은 재난대응 지식교육과 함께 개인의 흥미와 욕구를 반영함으 로써 자발적 학습동기를 유발하여야 함을 언급하고 있다. 또
한 행동은 학교재난안전 교육의 중요한 산출물로서 재난 대응 및 회복역량 등 재난안전의 실천적인 측면에 초점을 두고 있 다. 특히, 학생만을 대상으로 해서는 학교재난안전 교육의 목 적을 달성하기 어려우며, 학부모와 지역사회를 포함한 통합 적 관점에서 접근해야 한다고 강조하고 있다. 이들 주체의 재 난안전 교육의 자발적, 적극적 참여를 이끄는 방법으로 경쟁 (competitions), 시상(awards) 등을 통해 가정 및 지역사회의 흥미와 관심을 증진시키는 것이 효과적이며, 구체적인 방법으 로 사진, 미술 및 글쓰기 경진대회 등을 적극 활용할 것을 권고 하였다[13].

이러한 재난안전교육의 특성을 반영하여 UNISDR은 "학 교재난안전 교육은 교과서를 넘어서는 영역" 이라고 언급하면 서 흥미를 통한 비공식 재난안전교육을 강조하고, 공식적 교 육과정으로 융합할 것을 권고하고 있다. 아울러 학교재난안전 교육의 궁극적인 목적은 학생들을 재난위험저감을 위한 능동 적 주체로 육성하여 재난안전문화를 지역사회에 정착시켜 대 응 및 회복 역량을 강화시켜야 한다고 강조하였다[11].

\section{UN 학교재난안전 접근의 특징}

\section{1) 모든 위험사건 접근(all-hazards approach)}

최근 종래에 경험하지 못한 감염병, 테러 등 다양한 재난들 이 예측하기 어려운 형태로 빈번하게 발생하고 있다. 또한, 지 진, 쓰나미, 원전 폭발, 사회 혼란 등이 연쇄적으로 발생한 2011 년 동일본 대지진과 같은 복합재난도 향후 발생할 가능성이 높 다. 이러한 새로운 형태의 예측하기 어려운 현대 재난에 효과 적으로 대응하기 위해서는 종전의 특정 재난상황별 접근방법 보다 다양한 재난 형태에 공통적으로 적용할 수 있는 "모든 위 험사건 접근”이 효율적임을 밝히고 있다[14]. “모든 위험사건 접근”은 특정한 재난 대응에 요구되는 기능이 다른 유형의 재 난 대응에도 필요하며, 다양한 재난의 형태에도 불구하고 공통 된 대응 요소가 있다는 관점을 반영하고 있다. 나아가 Canton [15]은 "모든 위험사건 접근방법이 모든 대응책을 계획하는 것 은 아니다"라고 언급하면서 다음의 2가지를 “모든 위험사건 접 근방법”의 핵심요소로 제시하였다: 1) 다양한 재난의 주요 요 인인 지역사회 취약성에 대한 위험분석(risk analysis)에 초점 을 두고 있다; 2) 제한된 자원으로 효과적 대응을 위해서는 재난 경고 등 준비, 대피 및 피난, 회복 등 공통 핵심기능에 초점을 두 어야 한다는 것이다. 이러한 관점에서 “모든 위험사건 접근”은 지역사회의 모든 잠재적 재난 위험요소를 면밀히 규명하고, 발 생가능성 및 영향 등을 과학적으로 분석하며, 모든 재난 대응의 
공통핵심요소에 대하여 중점적으로 접근하는 방법으로 정의할 수 있다.

Stephens [16]은 점차 증가하고 있는 다양한 재난에 대한 효 과적 대응을 위하여 “모든 위험사건 접근”을 강조하고, 다음의 핵심적인 3 가지 공통 실천행동을 제시하고 있다: 1) 모든 재난 발생을 대비하여 비상물품키트를 준비하라; 2) 재난 발생 시 가족 간 재결합 장소 등 연락방안을 미리 마련해라; 3) 방송 등 을 통해 현재 재난 진행 사항에 대한 정확한 정보를 알고 있어야 한다. 이러한 관점은 감염병 대응에도 반영되어 $\mathrm{WHO}$ "보건비 상위험관리(Emergency Risk Management for Health)”의 핵심전략으로 최근 각국의 감염병 대책에 근간을 이루고 있다.

$\mathrm{UN}$ 은 “모든 위험사건 접근"을 학교재난안전에도 적용해 야 한다고 권고하고 있는 바, 학교 구성원·지역사회가 참여하 여 학교 및 지역사회의 모든 잠재적 재난위험요소를 규명하고 이를 제거하기 위한 다중적인 자원을 지속적으로 지원하여야 학교재난안전의 목적을 달성할 수 있다고 언급하였다. 최근 “모든 위험사건 접근”은 많은 국가의 학교재난안전에 영향을 미치고 있다. 미국의 경우 학교재난안전사고에 효과적으로 대응하기 위해 “모든 위험사건 접근”을 적용하여 2013년 “학 교위기대응계획 수립을 위한 안내(Guide for Developing High-Quality School Emergency Operations Plans, School $\mathrm{EOP})$ "를 수립하여 학교현장에 보급하였다. 이 지침은 빈번히 발생하는 학교 내 대형 총기사고를 포함하여 화재, 허리케인, 지진, 전염병 등 다양한 재난 위험을 철저히 규명하고, 이에 대 응함에 있어 통합된 계획 수립 및 공통 절차를 적용토록 하며, 재난 발생 전, 발생, 발생 후 등 3 단계로 구분하여 재난 유형별 로 차별화된 단계별 대응 매뉴얼을 제시하고 있다[17]. 일본의 경우 동일본 대지진을 겪은 후, 2012년에서 2016년까지 달성할 5 개년 계획으로 “모든 위험사건 접근” 을 적용한 “학교안전증 진계획"을 수립하고, 복합재난 등 새로운 재난 형태에 대비하 기 위하여 학생의 통합적 안전 능력을 향상시킬 수 있도록 학교 통합안전관리 계획 및 매뉴얼을 학교현장에서 의무적으로 수 립하도록 하였다[18].

\section{2) 지역사회 기반 접근}

재난안전에 대한 지역사회 기반 접근방법은 "지역사회의 취약 성 감소 및 재난역량을 제고시키기 위하여 재난위험의 규명·분석 · 관리 · 모니터링 - 평가 단계 등에 적극적으로 개입하는 전략"으 로 간략히 정의할 수 있다. 지역사회 기반 접근은 "통합위험관리 (comprehensive risk management)"의 실천전략으로 국가 차원에서 시작하는 하향식 접근보다는 지역사회 차원에서
출발하는 상향식 접근이 보다 효과적이라는 관점을 반영하고 있다[19]. 이와 유사한 접근 방법으로 Lindberg와 Sundelius [20]는 “전체사회(A Whole of Society)" 접근방법을 제시하고, 지역사회의 재난 회복탄력성을 핵심가치로 설정하고, 지역사회 의 두 가지 핵심 역량으로 “재난저항력(withstand)"와 “재난복 구력(bounce back)"을 강조하였다. 이를 위한 실천전략으로는 다양한 지역사회체계 간 네트워킹, 재난 발생 시 핵심 공공서 비스의 지속적 제공 계획, 다양한 지역사회체계가 참여하는 재난 시뮬레이션을 통한 학습, 커뮤니케이션 전략, 재정지원 의 혁신 등을 언급하였다.

이러한 재난안전에 있어 지역사회의 중요성은 총 6,400 명 의 사망자를 발생시킨 1995년 코베 대지진의 다음 교훈에서 명확히 드러나고 있다: 1) 대피할 시간을 거의 주지 않아 $90 \%$ 이상의 사망자가 발생 후 15 분 이내로 사망하였다; 2) 구조된 사람의 $80 \%$ 이상은 구조팀이 아닌 이웃 주민들에 의해 생명을 구할 수 있었다[21]. 이러한 교훈은 30년간 지진발생 사망원인 을 분석한 Bilham [22]의 연구결과에서도 나타나는 바, $83 \%$ 희생자가 사건발생과 함께 목숨을 잃기 때문에 중앙 차원보다 는 지역사회 재난안전이 중요하다고 언급하였다. 이러한 관점 에서 UNISDR은 국가적 차원의 많은 위기 대응 프로그램은 대부분이 단기간의 시행에 그쳐 지속적인 효과를 낳지 못하고 있음을 지적하고, 반드시 지역사회에 기반을 두고 장기적으로 시행되어야 한다고 강조하였다. 이를 위해 중앙정부의 지원과 지역사회의 참여가 긴밀히 연계되는 “지역기반 재난위험관리 프로그램(community-based disaster risk management)" 을 도입해야 한다고 언급하였다.

이러한 지역기반 재난위험관리는 학교재난안전에도 적용 되어, 각국의 학교재난안전정책에 있어 지역사회의 통합적 참 여는 핵심요소로 자리 잡고 있다. UNISDR의 "회복탄력적인 도시 만들기(making cities resilient)"캠페인은 지역사회의 재난 대응 및 회복역량을 강화하기 위한 10 개 요소 가운데 학 교 및 지역사회의 재난위험저감 교육 및 훈련 프로그램을 필수 요소로 제시하고 있다. 2013년 미국의 "학교위기대응계획 수 립을 위한 안내"에서도 위기계획 수립, 실행, 평가에 이르기까 지 지역사회가 반드시 참여해야 한다는 원칙을 제시하고 있 다. 또한 2011년 동일본대지진을 경험한 일본은 재난을 극복 하기 위한 교육개혁을 2013년 추진한 바, 4 대 기본방향 중의 하나로 “지역사회의 협력 및 결속”을 설정하여, 대지진 당시 지역사회의 역할이 무엇보다 중요하다는 교훈을 학교재난안 전에 반영하였다. 최근 UNISDR은 재난안전을 위해 지역사회 자원 활용의 중요성을 강조하면서 과학적 지식 이외에도 지역 
의 전통지식을 활용해야 함을 언급하고, "생존을 위한 지식 (Knowledge For Life)"이 되어야 함을 강조하였다[23].

이상에서 살펴본 바와 같이, $\mathrm{UN}$ 의 학교재난안전에서의 지 역기반 접근은 학교재난안전 계획 수립부터 평가 단계에 이르 기까지 지역사회가 통합적으로 참여함으로써 지역사회 요구 를 반영하고, 지역사회 자원을 활용하며, 나아가 지역사회 재 난안전문화 확산을 도모하는데 초점을 두고 있다. UN이 강조 하는 지역사회 기반 학교재난안전의 핵심 요소를 정리하면 다 음과 같다: 1) 학교의 지역사회 재난안전문화 확산 기여; 2 ) 학 교재난안전계획 수립 및 교육에의 지역사회 적극적 참여; 3 ) 지역사회 참여를 통한 학교위험 요소·취약성·대응능력 측정 및 평가; 4) 학교재난안전교육의 지속적 시행을 위한 지역사회 인적 ·물적 자원 지원; 5) 장애인 등 취약계층을 포함한 지역사 회의 재난안전 요구 반영; 6) 지역사회 재난안전교육 프로그램 마련; 7) 학교재난안전교육을 위한 지역사회의 지식 및 자원 활용 등.

\section{3) 지속발전 차원의 접근}

1987년 UN 환경개발세계회의에서는 지속발전을 "미래 세 대의 희생이 없는 범위에서 현재 필요를 충족하기 위한 발전" 으로 정의하고, 각국의 정치, 경제, 사회, 환경 및 교육정책 목 표에 “지속발전(sustainable development)”을 포함시킬 것 을 권고하였다. 또한 지속발전의 기본요소로 환경, 사회, 문화 및 경제 등 4 가지를 제시하고, 삶의 질 향상을 위해서는 이들 요소에 대한 지속적, 균형적인 고려가 있어야 함을 강조하였다. 이후 1992년 UN 환경개발세계회의 “Agenda 21"에서 “지속발전을 위한 교육(education for sustainable development)"의 개념을 도입하여, 재난 준비에서 회복 단계의 전 과정에서 환경·사회 · 경제적 취약요소의 제거를 통한 지속발전을 학교재난안전교 육의 목적으로 설정해야 한다고 언급하고 있다. 또한 지속발 전교육의 4 가지 요소로 1) 기초 교육 강화, 2) 학생 및 성인 시 민교육 실시, 3) 실천을 위한 교육훈련 강화, 4) 지속발전교육 체제로의 혁신 등을 제시하였다. 특히, 지속발전에서 요구하 는 기초 교육은 $3 \mathrm{R}$ 위주의 단순한 기능 증진을 의미하는 것이 아니라, 지역사회의 문제해결을 지원하고, 태도 및 가치 등을 증진시키는 교육을 의미하고, 실제 행동을 통한 학습을 의미 한다. 이러한 동향을 반영하여 UNESCO는 2005 2014년의 10 년간을 "지속발전을 위한 교육기간(the UN Decade of Education for Sustainable Development)"으로 정하고, 학 교교육에 지속발전의 개념을 통합시키기 위한 노력을 지속적 으로 추진함으로써 지속발전은 이미 많은 국가의 학교 교육
을 비롯하여 학교재난안전 비전으로 자리 잡고 있다. 나아가, INEE [12]는 지역사회 및 국가의 지속발전을 위해서는 재난 발생 시에도 학교교육이 중단되지 않고, 계속되어야 함을 강 조하고, “재난준비· 대응- 회복을 위한 교육최소기준”에 따라 “질 높은 교육(quality education)”을 제공해야 한다고 언급 하였다.

최근 UN의 지속발전교육의 개념은 각국의 학교안전 교육 과정에 반영되고 있는 추세이다. 미국의 학교건강안전모델 "모든 학교, 모든 학생, 모든 지역사회(Whole School, Whole Child, \& Whole Community)", 호주의 안전하고 지원적인 학교모델 "국가안전학교모형(National Safe Schools Framework)" 및 영국의 건강안전교육과정 "개인· 사회· 건강 및 경제 교육과정(Personal, Social, Health and Economic education)" 등은 근원적인 학생안전 증진을 위해서는 기초교육과 민주시 민교육에 초점을 두어야 한다는 지속발전교육의 관점을 반영 하고 있다. 특히, 일본의 경우에는 잦은 재난으로부터 학생안 전을 강화하기 위해 근본적인 교육과정 변화가 필요하다는 판 단으로 UN의 지속발전의 개념을 수용하였다. 이에 따라 2012 년 “학교안전증진계획”을 마련하고, “지속발전 사회를 위한 학교안전”을 새로운 학교안전정책의 비전으로 제시하였다. 후속 조치로서 2013년 추진한 학교교육개혁은 “생존을 위한 사회적 역량 개발”을 기본 방향으로 설정하고, 학생의 “삶의 열정(Zest for life)", 독립심 및 협동정신을 주요내용으로 포 함하여 스스로 배우고, 생각하며, 행동하는 문제해결능력을 강조하였다[18]. 이와 같이 각국의 학교재난안전 교육은 지속 발전을 이루는 핵심 기능으로, 정규 학교교육과정에 통합되고 있는 추세이다.

\section{4) 비공식 재난안전교육의 강조}

학교교육은 크게 공식교육과 비공식교육으로 구분할 수 있 으며, 공식교육(formal education)은 학교교과를 통해 이루 어지는 교육으로 계획적이고 목적적인 교육이며, 비공식교육 (informal education)은 학교 교과교육이외의 활동에서 이루 어지는 비구조적이고, 의도되지 않은 학습을 의미한다[25]. UNISDR [26]는 공식교육 이외에도 비공식교육을 통해 학생 들이 보다 많은 재난 정보를 학습해야 재난 대응능력을 향상시 킬 수 있다고 언급하고, 위험 학습은 빠를수록 효과적이기에 재 난안전에 있어 학교교육 이전단계의 비공식교육이 요구된다고 강조하였다. 지역기반 비공식교육의 대표적인 사례로 지역위 험지도(community risk map), 지역 관찰(town watching), 지역전문가 경험담 듣기, 지역체험학습 등을 제시하고, 이를 통 
해 학생들은 스스로 협동 학습을 통해 지역별로 발생 가능성이 높은 재난을 미리 파악하고, 대응방안 등을 토의함으로써 자연 스럽게 재난의식 및 대응능력을 함양시킬 수 있음을 제시하고 있다. 또한 Petal과 Izadkhah [27]은 지역사회와 함께 하는 재 난안전 활동을 중시하여 운동회, 전시회, 거리 공연 등을 활용 할 것을 언급하고, 재난안전의 중요성을 확산시키기 위해 미디 어를 통한 안전 관련 경연대회 및 캠페인 등이 효과적인 전략이 라고 강조하였다. 나아가 비공식적 재난안전 프로그램은 학생 들의 문제해결 능력을 개발하도록 하며, 재난을 올바로 이해하 고 대처하도록 한다고 언급하였다.

한편, $\mathrm{UN}$ 은 학교 또는 정부 주도만으로는 학교재난안전의 목적 달성에 한계가 있음을 지적하고, 비공식교육을 제공할 수 있는 보건기관, 민간기업, NGO 및 언론기관 등과의 연계를 강조하고 있다. 일례로 일본, 미국 등 선진국은 민간이 적극 참 여하여 다양한 재난 학습자료 및 프로그램 등을 마련하고 있 다. 일본 보험회사들이 제작한 “Inamura no Hi”와 같은 전래 동화를 활용한 재난안전 교재, 디즈니사가 후원하여 미국 적 십자사가 개발한 최초의 재난안전교육 모바일 게임인 "Monster Guard"등은 민간 기업이 재난안전 교육자료 개발- 보급에 적극 참여한 우수사례로 손꼽히고 있다.

최근 게임학습의 효과에 대한 연구가 증가하면서 재난안전 교육에 있어 적용사례가 증가하고 있다. Gee [28]는 학교에서 학습자의 공동체 문화를 반영한 게임을 통해 학습성과를 높일 수 있다고 밝혔다. 이와 같이 게임은 학습동기를 반영한 교육 환경을 제공함으로서 학습자간의 상호작용을 통하여 단순히 지식을 기억하는 수준을 넘어 보다 효과적인 이해 학습이 이루 어지도록 한다. 나아가, Lin 등[29]은 게임을 활용한 재난교육 은 효과적인 재난지식 전달과 함께 재난위험 저감을 위한 협동 관계를 증진시킬 수 있어 학교교육에서 적극 활용할 것을 권장 하였다. 또한 게임 학습과정은 게임 활동, 토론, 자기학습 등의 순으로 진행함으로써 학생들이 게임을 한 후 토론 시간을 통해 게임 중 자신의 행동과 생각을 돌이켜볼 기회를 제공하며, 토 론 후에는 전자책 등을 통해 자기학습을 하면서 재난 지식을 쌓도록 해야 한다고 제시하였다. 이러한 관점을 반영하여 UNISDR이 개발한 재난게임 "Stop Disasters"는 교사와 학 생 또는 학생 간 상호 작용을 유도하여 문제해결능력을 자발적 으로 증진시킨다는 평가를 받고 있으며, 세계 각국에 많은 영 향을 미치고 있다. 대표적인 사례로 호주의 "Dingo Creek"게 임은 학교 수업시간에 게임과정 및 결과에 대한 토론을 통해 재난 문제해결 및 대응역량을 강화시키고 있으며, 온라인을 통해 모든 학생이 다운로드 받을 수 있으며 정교하게 설계된
교사 수업용 안내서도 보급하고 있다.

나아가, $\mathrm{UN}$ 은 비공식 재난안전교육을 학교 정규교육과정 의 영역으로 흡수하는 교육과정의 융합을 강조하고 있으며, 필리핀 등 자연재해가 빈번한 일부 국가에서 도입을 추진하고 있다[30]. UNISDR는 재난안전의 교육과정 융합은 다음 과정 을 거쳐 수립되어야 한다고 언급하고 있다: 1) 학생 - 교직원 · 지역사회가 참여하여 재난위험 저감을 위한 핵심과제를 도출 한다; 2) 과제별로 필요한 재난 지식, 능력 및 기술의 영역과 순 서를 정교하게 기술한다; 3$)$ 수업계획에 통합 가능한 재난안전 내용이 무엇인지 교육과정을 면밀히 분석한다; 4 ) 비공식교육 의 학교교육과정 통합을 위한 교육 자료와 방법을 개발한다; 5) 교사 및 관련 교직원에게 교육자료 및 방법에 관한 연수를 시킨다; 6) 교사들에게 수업용 교재와 원격교육수단을 제공한 다; 7) 융합교육과정의 효과를 평가하고 조정-지원한다. 특히, 이와 같은 융합교육과정 마련은 재난안전의 복합적인 특성으 로 인해 5 년 이상의 장기간이 소요될 수 있어 지속적인 정책 지 원이 필요함을 강조하고 있다[27].

\section{결론 및 시사점}

최근 대형화, 복합화 되고 있는 재난은 막대한 인명 피해 및 경제적 - 사회적 손실 등을 초래하여 각국은 $\mathrm{UN}$ 의 권고를 수 용하여 재난위험저감 및 피해 감소를 위한 효과적 대응방안 수 립에 국가적인 역량을 집약시키고 있다. 우리나라의 경우에도 최근 몇 년간 세월호, 신종 감염병 등 예측하지 못한 인적·자 연적 재난의 연속적인 발생으로 전면적인 국가 재난안전 관리 체계의 개선이 이루어지면서 학교재난안전에도 큰 변화가 일 어나고 있다. 그러나 방대한 재난안전의 영역을 어떻게 다룰 것인가 등을 둘러싼 논란으로 많은 해결 과제들이 산적하여 있 는 상황이다. 이러한 시점에서 UN의 학교재난안전의 방향 및 주요내용을 비롯하여 이를 반영한 각국의 최근 동향들은 우리 나라 학교재난안전교육에 다음과 같은 의미 있는 시사를 주고 있다.

첫째, 학교재난안전 계획 수립에 있어 모든 위험사건 접근 방법을 적용하여야 할 것이다. 우선 학교의 모든 잠재적 재난 위험요소를 분석하고 효과적 재난 대응을 위한 공통요소를 추 출하여야 한다. 이를 반영하여 재난발생 단계별로 공통 대응 원칙 및 방안을 수립하여 어떠한 재난에도 대응할 수 있는 역 량을 증진함과 아울러, 지진 등 특정 재난 발생 시 효과적인 대 응 행동을 포함하는 통합적 학교 재난안전계획을 수립하여야 할 것이다. 
둘째, $\mathrm{UN}$ 은 효과적인 학교재난안전을 위해서는 학교와 지 역사회가 동반자적 자세로 학교재난안전계획 수립 단계부터 협력하여야 함을 강조하고 있으며, 이미 선진국 학교현장에서 는 보편화되고 있다. 그러나 우리나라는 학교재난안전계획 수 립 및 교육에의 지역사회 참여가 선진국에 비하여 현저하게 미 흡한 실정으로 지역사회 내 축적된 지식과 전문 인력을 활용하 여 위험요소를 규명하고, 지역 파트너들과 협력하여 해결하는 지역사회기반 학교재난안전 접근이 절실한 시점이다.

셋째, 학교재난안전교육에 있어 체험중심의 비공식교육을 강화하고, 효과적인 학습 자료 및 방법을 개발하여야 한다. 재 난안전교육에 있어 비공식교육은 공식교육에 못지않게 중요 하여 다양한 체험중심의 재난안전 교육기회를 제공해야 한다. 이를 위하여 부족한 안전체험교육관의 확충과 함께, 소방기관 등 기존 지역사회 기반시설의 인적·물적 자원을 최대한 활용 하여 체험학습 기회를 확대할 필요가 있다. 나아가 비공식 재 난안전교육을 공식교육에 융합하여야 한다. 비공식 재난안전 교육의 학교교육과정으로의 융합은 교육과정 수립 및 시행의 문제점 등에 대한 면밀한 검토가 필요하여 정부의 지속적인 관 심과 지원이 요구된다.

넷째, 학교재난안전교육의 비전과 목적을 명확히 설정해야 한다. 일본은 2011년 대지진을 경험한 후, UN의 권고를 수용 하여 학교안전의 비전으로 "지속발전을 위하여 스스로 생존할 수 있는 능력을 가진 독립심과 협동심을 갖춘 인간으로 육성 한다"라는 실천적인 방향을 제시하고 학교안전교육을 전면적 으로 혁신하고 있는 사례를 벤치마킹할 필요가 있다. 우리나 라의 경우에도 2014년 세월호 참사로 학생 개개인의 재난 대 응능력이 얼마나 중요한가를 깨닫는 계기가 되었지만, 여전히 재난안전 역량 교육이 학교교육에 정착되지 못하고 있는 실정 이다. 어떠한 재난 상황이 닥치더라도 학생 스스로 안전을 도 모할 수 있는 주체로 육성하고, 나아가 재난안전을 지속발전 차원에서 다루는 새로운 학교재난안전교육의 비전을 제시할 시점이라 할 것이다. 국가적으로 안전의 대변화가 일어나고 있는 상황에서 UN의 “모든 재난안전은 학교로부터”라는 기 치는 우리나라 재난안전교육의 기본방향이 되어야 할 것이다.

\section{REFERENCES}

1. Vanderveken A. The EM-DAT higher resolution disaster data [Internet]. Brussels: CRED; 2016 [cited 2016 October 9]. Available from: http://cred.be/sites/default/files/CredCrunch43.pdf

2. United Nations, International Strategy for Disaster Reduction. Towards a Culture of Prevention: Disaster Risk Reduction
Begins a School [Internet]. Geneva: UN/ISDR; 2007 [cited 2016 October 18]. Available from:

http://www.unisdr.org/files/761_education-good-practices. pdf

3. World Health Organization, The Emergency and Humanitarian Action. Disaster \& Emergencies Definitions [Internet]. Addis Ababa: WHO/EHA ; 2002 [cited 2016 October 21]. Available from: http://apps.who.int/disasters/repo/7656.pdf

4. Alexander D. The study of natural disasters, 1977-1997: some reflection on a changing field of knowledge. Disasters. 1997; 21(4):284-304. http://dx.doi.org/10.1111/1467-7717.00064

5. Basher R. Global early warning systems for natural hazards: systematic and people-centred. Philosophical Transactions of The Royal Society. 2006;364:2167-2182.

http://dx.doi.org/10.1098/rsta.2006.1819

6. Ansell J, Wharton F. Risk: analysis, assessment, management. First edition. New Jersey: Wiley; 1992. p. 5-98

7. United Nations International Strategy for Disaster Reduction. Hyogo framework for action 2005-2015: building the resilience of nations and communities to disasters [Internet]. Geneva: UNISDR; 2005. [cited 2016 October 15]. Available from: http://www.unisdr.org/files/1037_hyogoframeworkforactio nenglish.pdf

8. United Nations. Accelerating and main streaming disaster risk reduction efforts on the United Nations intensified approach [Internet]. Geneva: UNISDR; 2015 [cited 2016 October 15]. Available from:

http://www.unisdr.org/files/45550_acceleratingmainstream ingdrrefforts.pdf.

9. Prior T, Roth F. Global Disaster Politics Post Sendai. CSS Analyses in Security Policy. 2015 May 1:1-4.

10. The United Nations Children's Fund, the Inter-agency Network for Education in Emergencies, The International Federation of Red Cross, The United Nations Educational, Scientific and Cultural Organization, World Vision, et al. Comprehensive School Safety [Internet]. New York: UNICEF; 2013 [cited 2016 October 11]. Available from: http://www.unesco.org/new/fileadmin/MULTIMEDIA/HQ /SC/pdf/Comprehensive_school_safety.pdf

11. Petal M. Disaster Prevention for Schools Guidance for Education Sector Decision-Makers Consultation version [Internet]. Geneva: UNISDR; 2008 [cited 2016 October 20]. Available from: https://www.unisdr.org/we/inform/publications/7556

12. the Inter-agency Network for Education in Emergencies. Minimum standards for education: Preparedness, response, recovery [Internet]. New York: IMEE; 2010 [cited 2016 October 11]. Available from:

http://toolkit.ineesite.org/toolkit/INEEcms/uploads/1012/ INEE_GuideBook_EN_2012\%20LoRes.pdf

13. Shaw R, Takeuchi Y, Shiwaku K, Fernandez G, Gwee QR, Yang 
B. 1-2-3 of disaster education [Internet]. Kyoto: European Union, ISDR, Kyoto University; 2009 [cited 2016 October 25]. Available from: http://www.unisdr.org/files/12088_123sm.pdf

14. World Health Organization. Risk reduction and emergency preparedness [Internet]. Geneva: WHO; 2007 [cited 2016. October 22]. Available from:

http://www.who.int/hac/techguidance/preparedness/eme rgency_preparedness_eng.pdf

15. Canton LG. "All-hazards" Doesn't Mean "Plan for Everything" All-hazards planning requires a risk-based approach [Internet]. Emergency Management; 2013 [cited 2016 October 26]. Available from:

http://www.emergencymgmt.com/emergency-blogs/manag ing-crisis/Allhazards-Doesnt-Mean-Plan-for-Everything.html

16. Stephens KM. All Hazards Preparedness Guide [Internet]. Atlanta: CDC; 2013 [cited 2016 October 12]. Available from: https://www.cdc.gov/phpr/documents/ahpg_final_march_ 2013.pdf

17. U.S. Department of Education, U.S. Department of Health and Human Services, U.S. Department of Homeland Security, U.S. Department of Justice, Federal Bureau of Investigation, Federal Emergency Management Agency. Guide for Developing HighQuality School Emergency Operations Plans [Internet]. Washington: U.S. Department of Education; 2013 [cited 2016 October 19]. Available from:

http://rems.ed.gov/docs/REMS_k-12_Guide_508.pdf

18. Ministry of Education, Culture, Sports, Science and Technology. The second basic plan for promotion of education [Internet]. Tokyo: Government of Japan MEXT; 2013 [cited 2016 October 16]. Available from:

http://www.mext.go.jp/a_menu/keikaku/detail/_icsFiles /afieldfile/2013/06/20/1336379_01_1.pdf.

19. Kafle SK, Murshed Z. Community-based disaster risk management for local authorities [Internet]. Klong Luang Pathumthani, Thailand: Asian Disaster Preparedness Center; 2006 [cited 2016 October 22]. Available from: http://www.unisdr.org/files/3366_3366CBDRMShesh.pdf

20. Lindberg H, Sundelius B. Whole-of-Society Disaster Resilience. In: Kamien D, editor(s). The McGraw-Hill Homeland Security Handbook: Strategic guidancce for a coordinatated approach to effective security and emergency management. Second edition. Columbus: The McGraw-Hill Homeland Companies; 2012. p. $1295-1297$.

21. Suganuma K. Recent Trends in Earthquake Disaster Management in Japan. Quarterly Review. 2006 April 19:91-106

22. Bilham R. Lessons from the haiti earthquake. Nature International weekly journal of science 463. 2010 February 17:878-879.

23. The United Nations Office for Disaster Risk Reduction. Knowledge for Life: UN launches search for communities good at managing the risk of disasters. 2015 July 28:1.

24. Wals A. Review of contexts and structures for education for sustainable development [Internet]. Paris: UNESCO; 2009 [cited 2016 October 12]. Available from:

http://unesdoc.unesco.org/images/0018/001849/184944e.pdf

25. Scribner S, Cole M. Cognitive Consequences of Formal and Informal Education. Science. 1973;182:553-559.

26. International Strategy for Disaster Reduction. Leaders of Indonesia's Simeulue community received a prestigious UN award [Internet]. Bangkok: ISDR; 2005 [cited 2016 October 16]. Available from:

http://www.unisdr.org/files/5602_IndonesiacommunityUN award.pdf

27. Petal MA, Izadkhah YO. Formal and informal education for disaster risk reduction [Internet]. Islamabad: Risk RED; 2008 [cited 2016 October 14]. Available from: http://www.riskred.org/activities/ddredislamabad.pdf

28. Gee J P. What video games have to teach us about learning and literacy. New York: Palgrave Macmillian; 2003. p. 1-12.

29. Lin SC, Tsai MH, Chang YL, Kang SC. Game-initiated learning: A case study for disaster education research in Taiwan. 2013 Spring Symposium Series; 2013 March 25-27; Palo Alto California. Conference Paper 2013 AAAI Spring Symposium: Association for the Advancement of Artificial Intelligence; 2013. p. 67-71.

30. Selby D, Kagawa F. Disaster Risk Reduction in School Curricula: Case Studies from Thirty Countries [Internet]. Spain: UN ESCO, UNICEF; 2012 [cited 2016 October 26]. Available from: https://www.unicef.org/education/files/DRRinCurriculaMapping30countriesFINAL.pdf 